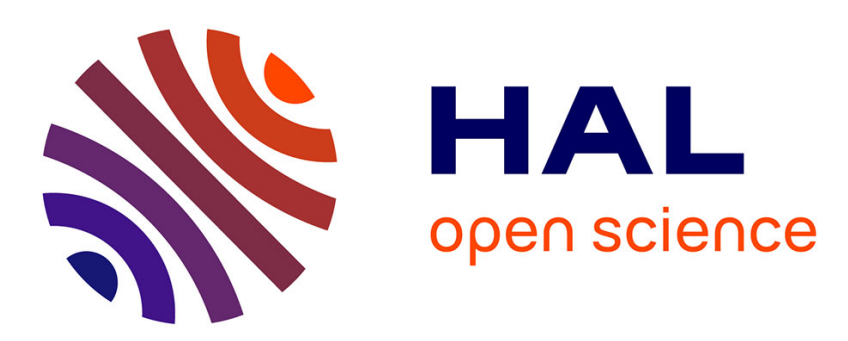

\title{
Polarization of electron-beam irradiated LDPE films: contribution to charge generation and transport
}

Mallys Elliazar Banda, Virginie Griseri, G. Teyssedre, Séverine Le Roy

\section{To cite this version:}

Mallys Elliazar Banda, Virginie Griseri, G. Teyssedre, Séverine Le Roy. Polarization of electronbeam irradiated LDPE films: contribution to charge generation and transport. Journal of Physics D: Applied Physics, 2018, 51 (15), pp.155303. 10.1088/1361-6463/aab467 . hal-02346717

\section{HAL Id: hal-02346717 https://hal.science/hal-02346717}

Submitted on 14 Dec 2020

HAL is a multi-disciplinary open access archive for the deposit and dissemination of scientific research documents, whether they are published or not. The documents may come from teaching and research institutions in France or abroad, or from public or private research centers.
L'archive ouverte pluridisciplinaire HAL, est destinée au dépôt et à la diffusion de documents scientifiques de niveau recherche, publiés ou non, émanant des établissements d'enseignement et de recherche français ou étrangers, des laboratoires publics ou privés. 


\title{
Polarization of electron-beam irradiated LDPE films: contribution to charge generation and transport
}

\author{
M E Banda, V Griseri, G Teyssèdre and S Le Roy \\ LAPLACE, Université de Toulouse, CNRS, INPT, UPS, France
}

\begin{abstract}
Electron-beam irradiation is an alternative way to generate charges in insulating materials, at controlled position and quantity, in order to monitor their behaviour as regard transport phenomena under the space charge induced electric field or external field applied. In this study, Low Density Polyethylene (LDPE) films were irradiated by a $80 \mathrm{keV}$ electron-beam with a flux of $1 \mathrm{nA} / \mathrm{cm}^{2}$ during 10 minutes in an irradiation chamber under vacuum conditions, and were then characterized outside the chamber using three experimental methods. The electrical behaviour of the irradiated material was assessed by space charge measurements using the Pulsed Electro-Acoustic (PEA) method under dc stress. The influence of the applied electric field polarity and amplitude has been tested in order to better understand the charge behaviour after electron-beam irradiation. Fourier Transform Infra-Red spectroscopy (FTIR) and Photoluminescence (PL) measurements were performed to evaluate the impact of the electron beam irradiation, i.e. deposited charges and energy, on the chemical structure of the irradiated samples. The present results show that the electrical behaviour in LDPE after irradiation is mostly driven by charges, i.e. by physical processes function of the electric field, and that changes in the chemical structure seems to be mild.
\end{abstract}

PACS Numbers: $72.20 \mathrm{Ht}, 72.20 \mathrm{Jv}, 77.22 \mathrm{Ch}$

Keywords-LDPE, Electron-beam irradiation, Space charge, FTIR, Photoluminescence, degradation.

\section{Introduction}

Whether for electronics, electrical engineering systems or space environment applications, polymeric materials are increasingly used as insulating materials. Under various electrical, thermal, mechanical or radiative constraints, these insulating materials can accumulate charges that can irreversibly affect their properties and harm the reliability of the depending systems [1,2]. Prediction of these damages implies a better understanding of the space charge generation and transport mechanisms in such material.

In order to achieve this aim, a common way consists in applying a DC voltage to an insulation sandwiched between two electrodes and to measure the space charge distribution in the material bulk. However, this technique is not sufficient to distinguish the contributions from charge generation and transport mechanisms. Charge deposition using an electron-beam source is an alternative technique to generate charges at a controlled location and quantity. Nevertheless, other physical processes take place during electron-beam irradiation, which provides ionizing radiation besides primary charges source. Positive/negative carrier creation, that is thought to take place during e-beam irradiation due to ionization $[3,4]$, is particularly difficult to highlight experimentally, as these pairs are located at the same place, leading to a net zero charge density. The application of a sufficiently high electric field in post-irradiation induces a movement of the positive/negative charges in opposite directions. It should uncover the positive and negative charges generated by the e-beam. This has already been performed in other studies for polymeric materials intended to space applications $[5,6]$. 
The aims of this paper is to verify if the creation of electron/hole pairs can be observed in particular postirradiation conditions, and to check if for our irradiation conditions, irreversible damages, i.e. ageing, take place, as e-beam irradiation is also thought to degrade the chemical structure of the insulation $[7,8]$. In doing so, we intend to assess the physical hypotheses that are linked to e-beam irradiation and charge transport in solid organic insulations. Low-density polyethylene (LDPE) has been chosen for this study, as it remains the most studied insulating material, owing to its simple chemical structure. After e-beam irradiation in a dedicated vacuum chamber (MATSPACE) [9], space charge measurements are performed ex-situ in a conventional space charge measurement set-up using the Pulsed Electro-Acoustic method (PEA), under applied dc voltage. The experimental profiles are compared to a reference nonirradiated LDPE sample, for the same experimental protocol. The influences of the electric field amplitude and polarity are presented and physical hypotheses are proposed to explain the space charge behaviour. Physico-chemical characterizations have been performed, to evaluate the impact of the ebeam on the chemical structure of the sample. At last, we discuss the most likely physical processes driving the peculiar behaviour of LDPE sample under applied voltage after e-beam irradiation.

\section{Material and measurement procedure}

\subsection{Sample preparation and irradiation conditions}

LDPE samples of thicknesses ranging from $250 \mu \mathrm{m}$ to $300 \mu \mathrm{m}$, and a diameter of $70 \mathrm{~mm}$ were prepared from pellets, using a hydraulic press and a specific mould. In the process, cover layers (made of Polyimide material -PI) are used to prevent the sample from sticking to the mould surface and to provide a smooth surface. The pellets are heated from room temperature up to $160^{\circ} \mathrm{C}$ at atmospheric pressure. A pressure of 2.5 bars is then applied on the sample during 25 minutes. The pressure and temperature are then smoothly decreased to room temperature and atmospheric pressure (around 45 min cooling time). The PI cover layers are then removed.

LDPE samples are then inserted in an irradiation chamber [9], under a vacuum of $\sim 10^{-7} \mathrm{mbar}$, and submitted to a $80 \mathrm{keV}$ electron-beam for 10 minutes with a current flux of $1 \mathrm{nA} / \mathrm{cm}^{2}$. Samples can be characterized in situ, i.e. under vacuum conditions, using the in situ Pulsed Electro-Acoustic (PEA) space charge set-up. They can also be removed from the irradiation chamber, i.e. they return to atmospheric pressure, and are characterized outside the chamber, using a 'classic' Pulsed Electro Acoustic (PEA) method, where a voltage can be applied. In this study, we mostly used this latter set-up for electrical characterization of the LDPE irradiated samples under atmospheric pressure.

\subsection{Space charge measurements under DC electric field}

Ex-situ, before voltage application, the irradiated sample is placed under short-circuit conditions during 5 minutes, and space charge measurements are performed, in order to check if the change from vacuum conditions to atmospheric pressure conditions does not affect the space charge distribution to a large extent.

A protocol of one hour polarization/one hour depolarization is then applied to the sample, with an electric field varying from 5 to $40 \mathrm{kV} / \mathrm{mm}$. For these measurements, the sample is directly in contact with the semiconducting top electrode of the PEA set-up, supplying the voltage, and with the aluminium bottom electrode, which is grounded. The electroacoustic signal from PEA response was obtained using pulses of $600 \mathrm{~V}$ in amplitude, generated from a $1 \mathrm{kHz}$ pulse generator. The calibration step is performed prior any irradiation in the case of irradiated samples. Space charge profiles were recorded every 30s. For each measurement, a new sample has been used to avoid sample history effects on the response. All measurements were realized at room temperature.

\subsection{FTIR and Photoluminescence measurements}

FTIR analyses were performed on a VERTEX 70 fully digital infra-red (IR) spectrometer designed for data acquisition in the mid IR region $\left(400-4000 \mathrm{~cm}^{-1}\right)$ with a resolution of $2 \mathrm{~cm}^{-1}$. The noise level was 
significantly reduced before any measurements by purging atmospheric gases (water vapour and carbon dioxide) using dry air and nitrogen gaz. Each experiment begins by acquiring the background spectrum without sample, in the purged compartment. The FTIR results were obtained in the same conditions for both the irradiated and the non-irradiated samples, in transmission mode.

Photoluminescence (PL) measurements were also performed on both the irradiated and the non-irradiated LDPE samples. Samples were mounted in a light-tight chamber used for multipurpose light detection from polymer materials, including electroluminescence measurements [10]. Three quartz windows allow integral/spectral light detection and photoluminescence excitation. IN PL, the wavelength resolved spectrum of the light emitted under excitation at selected wavelength by a Xenon lamp coupled to a monochromator was integrated using a liquid-nitrogen-cooled CCD camera and a dispersive system with a resolution of $4.5 \mathrm{~nm}$. The detection wavelength is in the range 250 to $842 \mathrm{~nm}$ and the excitation wavelength was varied between 220 and $300 \mathrm{~nm}$, with a bandwidth of the irradiation windows fixed at around $2 \mathrm{~nm}$. The excitation wavelength range (taken by step of $5 \mathrm{~nm}$ ), corresponding to the optical absorption range of the polymer unsaturation, was chosen in order to optimize the emission. The emission and detection system are coupled to the sample surface through quartz lenses.

\section{Experimental results and discussion}

\subsection{Reference measurement without irradiation}

A space charge measurement, which will serve as reference, was firstly performed on a virgin LDPE film of thickness $300 \mu \mathrm{m}$. The sample was polarized under a DC voltage of $+12 \mathrm{kV}$ applied at the semiconducting electrode (corresponding to an electric field of $40 \mathrm{kV} / \mathrm{mm}$ ) for $1 \mathrm{~h}$ and then shortcircuited for $1 \mathrm{~h}$, at $25^{\circ} \mathrm{C}$. Figure 1 shows the cartography, and its equivalent $2 \mathrm{D}$ representation, of the space charge distribution as a function of time in the case of a non-irradiated sample.
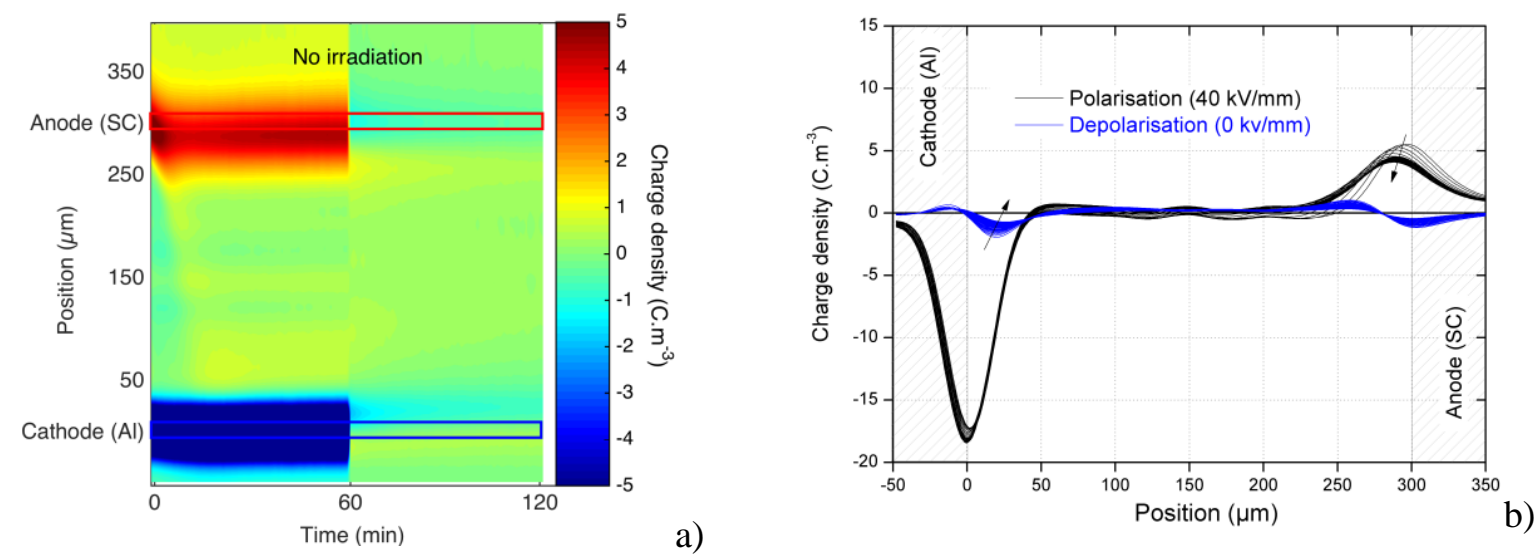

Figure 1. (a) Cartography and (b) Profiles of space charge density, taken each 1 min, for a nonirradiated LDPE sample of thickness $300 \mu \mathrm{m}$, under a voltage of $+12 \mathrm{kV}$ on the top electrode (i.e. electric field of $40 \mathrm{kV} / \mathrm{mm}$ ) for $1 \mathrm{~h}$ (black lines) and then short-circuited for $1 \mathrm{~h}$ (blue lines), at $25^{\circ} \mathrm{C}$.

During polarization, positive charges are detected, arising from injection at the anode (top electrode). These positive charges transport through the dielectric and cross the material in about 20 min. These positive charges seem to accumulate at the cathode with time. No negative charges are observed during volt-on measurements. When the sample is short-circuited, positive charges remain in the bulk, while negative charges are observed very close to the cathode. These negative charges have certainly been injected at the cathode (bottom electrode) during polarization but would not be observed at this time due to overlapping by capacitive charges and the presence of positive space charges.

This behaviour of space charge, regarding the dominance of positive charges and the transit time, is in agreement with the literature on LDPE $[11,12]$. The space charge density in the bulk of the non-irradiated sample remains low $\left(<0.5 \mathrm{C} / \mathrm{m}^{3}\right)$ during and after electric field application. 


\subsection{Space charge after electron-beam irradiation}

Calculations have been performed, using an open access Monte Carlo simulation code called PENELOPE [13], to predict the electron deposition profile for an electron beam of energy $80 \mathrm{keV}$ and a constant flux of $1 \mathrm{nA} / \mathrm{cm}^{2}$. This Monte Carlo code allows simulating the trajectory of a large number of particles over a wide energy range, accounting for the matter-radiation interactions. In our case, the particles are only electrons. The Monte Carlo code does not take into account the processes linked to conduction or to energy deposition. Figure 2 shows the calculated deposited electron density and the equivalent dose, as a function of the sample thickness for a $300 \mu \mathrm{m}$ thick LDPE. The maximum deposited dose and electron density are respectively located at around $45 \mu \mathrm{m}$ and $65 \mu \mathrm{m}$ from the irradiated face, while the irradiated region extends over around $100 \mu \mathrm{m}$.

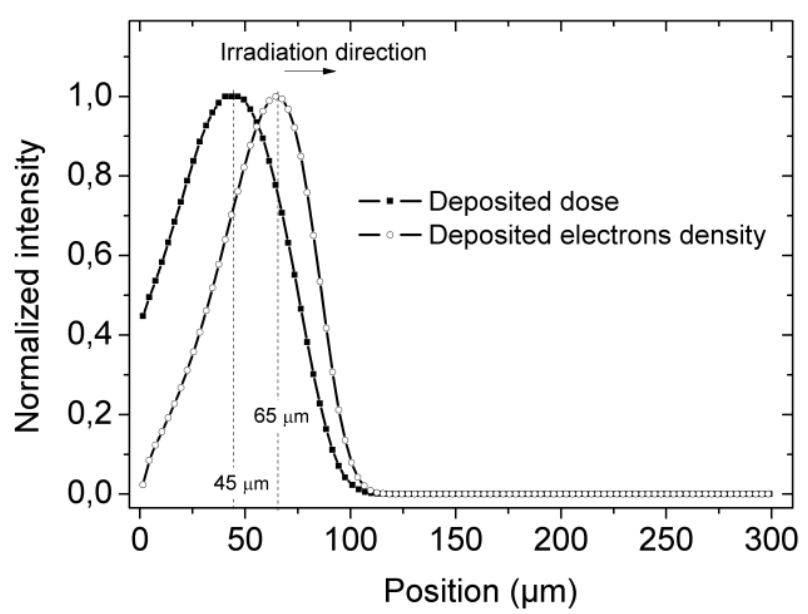

Figure 2. Normalized deposited dose and electron density due to the electron beam as a function of the position in the sample calculated by Penelope [13] for a $300 \mu \mathrm{m}$ thick LDPE irradiated with a $80 \mathrm{keV}$ electron beam.

Space charge measurements were then performed on similar LDPE films of thickness $300 \mu \mathrm{m}$, prepared with the same process and irradiated under vacuum with an electron beam of $80 \mathrm{keV}$ for 10 minutes with a constant flux of $1 \mathrm{nA} / \mathrm{cm}^{2}$.

In-situ PEA measurements using an 'open PEA' set-up, have been performed under vacuum during ebeam irradiation, in order to probe the charge build-up during irradiation [14] and compare to the theoretical calculations. The top electrode is at remote distance from the surface avoiding in this way discharge of the sample from the irradiated face. Figure 3 (black curve) shows the charge distribution in this case, the irradiated face being on the right side. A negative charge peak is observed at around $100 \mu \mathrm{m}$ from the irradiated face, with a maximal density of $-15 \mathrm{C} / \mathrm{m}^{3}$. The peak maximum is located deeper in the insulation compared to the theoretical calculations $(\sim 65 \mu \mathrm{m})$. This difference could come from the fact that the Monte Carlo code, dedicated to matter-radiation interactions, does not take into account specific processes such as transport of deposited charges and radiation-induced conductivity. 


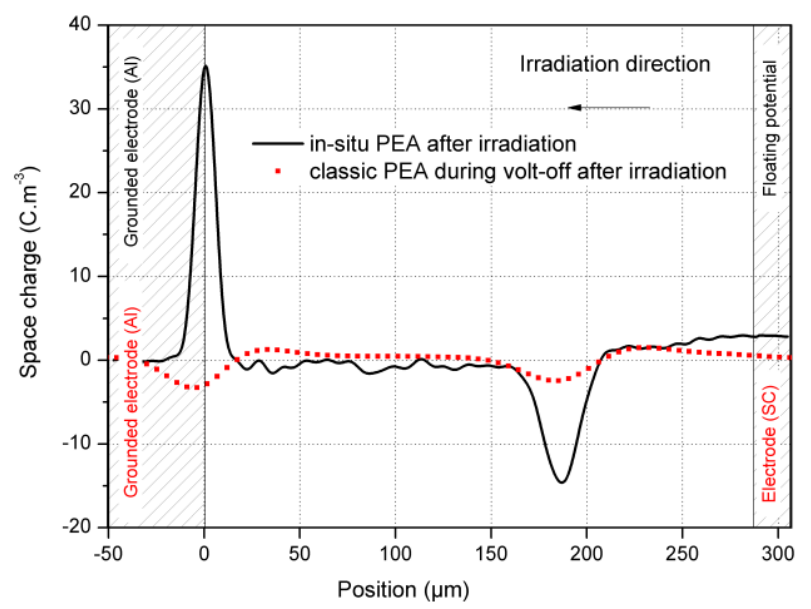

Figure 3. Comparison of the space charge density from an in-situ PEA set-up with a floating potential at the irradiated face, and a 'classic' PEA set-up during volt-off after irradiation (first minutes of figure 4). Irradiation comes from the right side for each measurement.

Figure 4 presents the space charge measurements performed with a 'classic' PEA set-up after e-beam irradiation on the same kind of LDPE sample of thickness $300 \mu \mathrm{m}$. The first 5 minutes of the experiment are under short-circuit conditions, in order to check the presence of deposited electrons. The presence of a peak of negative charges at $100 \mu \mathrm{m}$ from the irradiated face is consistent with in-situ PEA measurements. The maximum value of the charge density peak (figure 3 ) is however higher in the case of in-situ measurements $\left(-15 \mathrm{C} / \mathrm{m}^{3}\right)$ compared to the one observed in figure 4 during the first 5 minutes in volt-off $\left(\sim-3 \mathrm{C} / \mathrm{m}^{3}\right)$. Although the physics behind this is not definitively understood, this decrease of charge is mainly due to a change of environment from vacuum to air conditions.

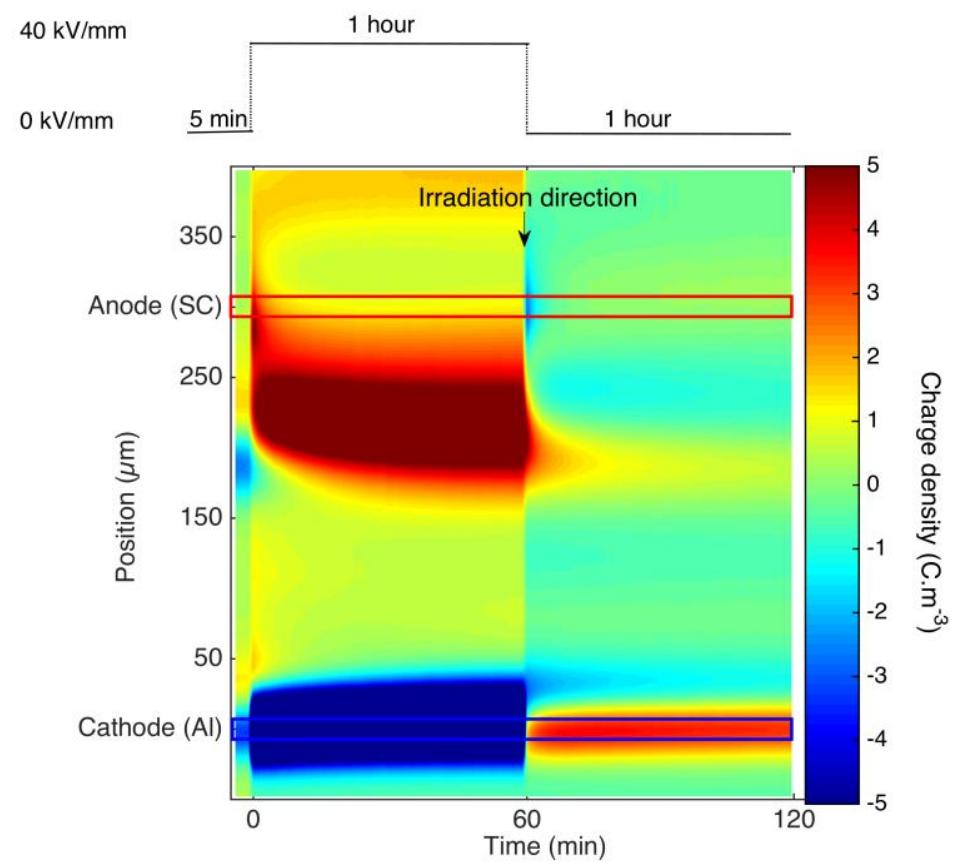

Figure 4. Cartography of space charge density for an e-beam irradiated LDPE film of $300 \mu \mathrm{m}$ thickness, at $25^{\circ} \mathrm{C}$. The sample was consecutively short-circuited for 5 minutes, polarized with $+12 \mathrm{kV}$ on the top electrode (i.e. electric field of $40 \mathrm{kV} / \mathrm{mm}$ ) for one hour and short-circuited for one hour. Irradiated zone next to the top electrode.

Surprisingly, positive charges are also observed before any voltage application. They are located at $65 \mu \mathrm{m}$ from the irradiated face, and also detected near the bottom electrode, at about $265 \mu \mathrm{m}$ from the 
irradiated face. These positive charges, present with a non-negligible density (above $1 \mathrm{C} / \mathrm{m}^{3}$ ), are not due to experimental artefact. The same features have already been reported, showing positive charges measured experimentally during relaxation $[5,15]$. Moreover, only a bipolar charge transport model (i.e. positive and negative charges) could reproduce these experimental results [16]. However, these positive charges have different origins, depending of their location.

Positive charges detected at $65 \mu \mathrm{m}$ from the irradiated face are probably due to the creation of positive/negative carrier pairs during irradiation, a physical process largely described in the literature [4, 17] but not often reported in experiments. These positive charges are certainly observed here due to variation of the electric field distribution in the sample from vacuum conditions in the irradiation chamber, i.e. floating potential at the irradiated face, to $0 \mathrm{~V}$ at the top electrode (being previously the irradiated face) during volt-off conditions. It is not clear whether these charges are positive ions or electronic carriers (i.e. holes). However, the literature on this subject often refers to electron/hole pair creation [17,18] or to the creation of positive mobile species [4], so we will keep the term of electron/hole pair generation. Indeed, this experimental observation reinforces the physical hypothesis of creation of electron/hole pairs during irradiation. On the contrary, positive charges at the bottom electrode should arise from charge injection during irradiation, as the sign and the value of the electric field during irradiation at the bottom electrode are favourable to positive charge injection.

After these 5 minutes time in volt-off, the sample was polarized under a DC voltage of $+12 \mathrm{kV}$ on the top electrode (corresponding to an electric field of $40 \mathrm{kV} / \mathrm{mm}$ ) during 1 hour followed by 1 hour under volt-off. As soon as the voltage is applied, a large amount of positive charges is measured in the whole irradiated zone, and remains stable until the end of the polarization time. The position of these positive charges never exceeds the limit of the irradiated zone (i.e. around $120 \mu \mathrm{m}$ from the irradiated face). The presence of negative charges deposited by the beam and previously observed during volt-off, is no more observable, as the amount of positive charges is very high. Negative charges are also detected at the bottom electrode, certainly generated by injection at the cathode. The comparison of charge density for an irradiated sample and a non-irradiated sample at the end of the polarization step is presented in figure 5. Although the dominant charges remain positive, the distribution is completely different. Compared to the reference sample, where positive charges are distributed in the whole insulation with a small density, the positive charges for the irradiated sample are mainly located in the irradiated zone, with a broad distribution.

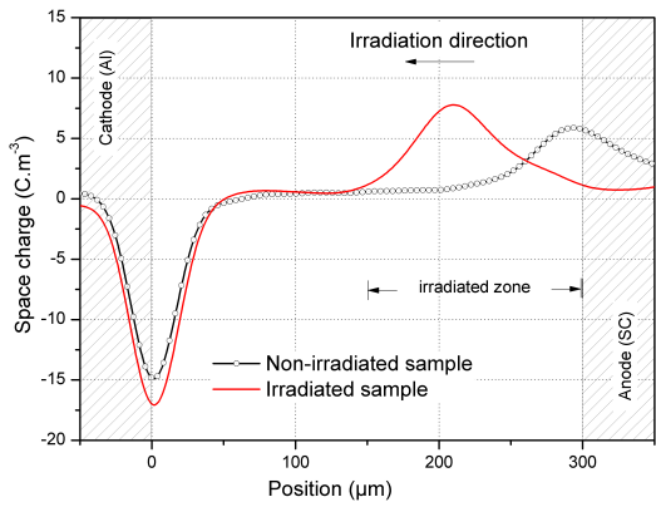

Figure 5. Comparison of the space charge density for the reference sample (i.e. non irradiated) and the irradiated sample, at the end of a polarization protocol of $40 \mathrm{kV} / \mathrm{mm}$. LDPE of thickness $300 \mu \mathrm{m}$. Anode on the right side, cathode on the left.

Immediately after voltage drop to zero, there is a fast decay of the positive charges density in the irradiated zone (cf. figure 4). These positive charges remain detectable only at the limit of the irradiated zone. Otherwise, negative charges appear in the dielectric during short-circuit. These charges are certainly already present during volt on, but hidden by the presence of positive charges in higher density. The space charge pattern during volt-off for an e-beam irradiated sample also differs from the reference one, as more negative charges are detected and the location of positive charges is slightly different.

Different processes could be at the origin of the large amount of positive charges in the irradiated zone: 
- Charge injection at the anode: the presence of negative charges and the application of a positive voltage at the top electrode enhance the electric field at this electrode, allowing a massive positive charge injection and then a transport of these charges within the bulk. However, one could wonder if the small amount of negative charges detected during the 5 min of short circuit before polarization is enough to increase the injection to a so large extent;

- Transport and extraction of electrons: during irradiation, primary electrons are deposited and electron/hole pairs are created. Charges in these pairs are of the same density, at the same location, and hence constitute a net zero charge density. However, as soon as a high electric field is applied, this equilibrium is disturbed and when electrons in the irradiated zone move and/or are extracted at the anode, a net positive charge density sets;

- The energy of the electron beam can also have an impact on the chemical structure of the dielectric. The irradiation could have changed the dielectric properties in the irradiated zone, such as mobility, compared to a non-irradiated material. This may induce a fast motion of positive charges.

The first two hypotheses are linked, as they are driven by the electric field enhancement due to the presence of charges. The third hypothesis is related to the changes in the chemical structure of the material. It is difficult to figure out a priori which scenario is likely to take place, all the more that measurements have been performed in order to segregate one process upon the other.

\subsection{Influence of the electric field polarity}

To check for injection/extraction effects on the burst of positive charges detected in the whole irradiated zone, complementary measurements were performed with a negative voltage stress on the top electrode, keeping the same experimental conditions, i.e. the irradiated zone is still located at the top electrode. Figure 6 shows the space charge map obtained with this change of sign of the electric field. The first 5 minutes of short-circuit at the beginning still ensure that the space charge profile at the end of the irradiation remains the same, using a new sample. As previously, the present sample shows the presence of negative charges located at around $100 \mu \mathrm{m}$ from the irradiated face, and positive charges next to each electrode.

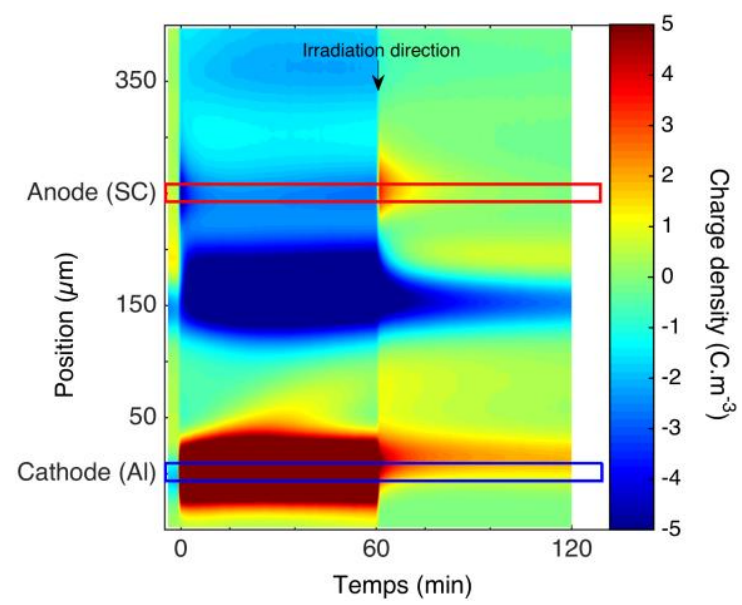

Figure 6. Cartography of space charge density for an e-beam irradiated LDPE film of $250 \mu \mathrm{m}$ thickness, at $25^{\circ} \mathrm{C}$. Short-circuit for 5 minutes; negative voltage applied on the top electrode resulting in an electric field of $40 \mathrm{kV} / \mathrm{mm}$ for one hour; and then short-circuit for one hour. Irradiated zone next to the top electrode.

Once the voltage is applied, a large amount of negative charges is instantaneously detected in the whole irradiated zone. The phenomenon seems even amplified compared to the previous section, as more negative charges seem to be present. Positive charges are also injected at the bottom electrode and transported through the bulk. During depolarization, the bulk of the material is slightly positively 
charged, and negative charges are detected only at the limit of the irradiated region. The charge density decreases quickly with time. Comparatively to the previous results in section 3.2, changing the electric field polarity only changes the sign of charges detected in the whole material, but no different physical phenomenon is observed. This is all the more surprising, as negative charges previously stored in the irradiated region should act against negative charge injection to the top electrode, the electric field being reduced by the presence of these negative charges. Moreover, positive and negative charges do not have the same mobility in LDPE at room temperature [19].

\subsection{Influence of the applied electric field amplitude}

Charge injection/extraction at the electrodes is function of the local electric field. The influence of the applied electric field amplitude was tested in order to evaluate its impact on the burst of charges at the anode. Figure 7 presents the space charge profiles for e-beam irradiated LDPE films (same conditions as described in section II) for applied fields of amplitude 40, 20, 10 and $5 \mathrm{kV} / \mathrm{mm}$ (a to d), a positive voltage being applied to the top electrode. For all applied electric fields, positive charge build up in the irradiated zone is observed, and the density is directly related to the field amplitude. The charge density in the irradiated zone decreases with the decrease of the applied field. However, for all cases, the positive charge appearance is rapid and the kinetics seems unchanged. Positive charge injection, if this hypothesis holds for the presence of a large amount of positive charge in the irradiated zone, should be function of the electric field, and should take more time to build when the electric field is lower. The same line of argument holds for electron extraction at the anode. Electron transport is also function of the electric field, and should take more time when the electric field is lower. Either the electric field due to charges implanted by the e-beam is high enough and the applied electric field is just a small perturbation of this field due to charge, or the charge behaviour is not driven by the electric field distribution. Electrons injection is also observed at the bottom electrode, mostly for an electric field of $40 \mathrm{kV} / \mathrm{mm}$. This is less the case for the other electric fields, which are too low to allow negative charges to be dominant next cathode region.
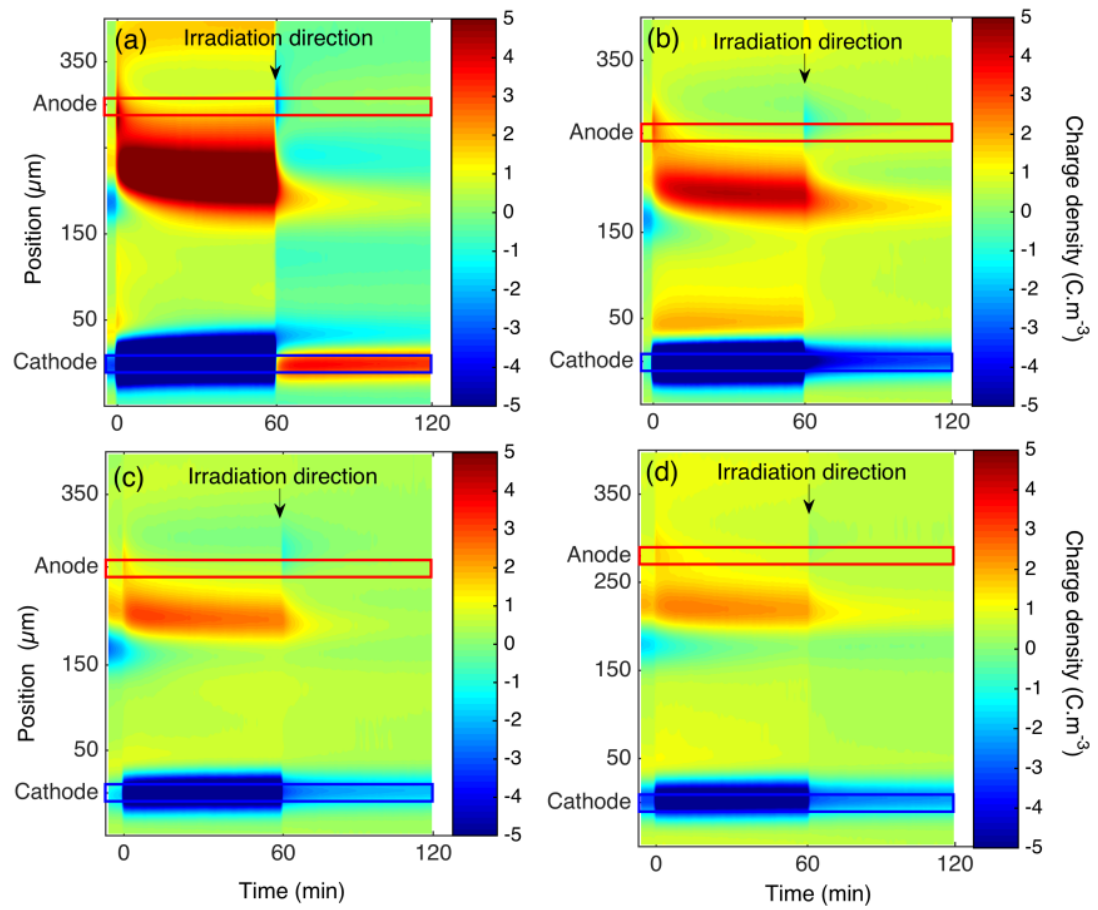

Figure 7. Cartography of space charge density for e-beam irradiated LDPE films under DC electric field of (a) $40 \mathrm{kV} / \mathrm{mm}$, (b) $20 \mathrm{kV} / \mathrm{mm}$, (c) $10 \mathrm{kV} / \mathrm{mm}$, (d) $5 \mathrm{kV} / \mathrm{mm}$. The irradiated zone is always next to the top electrode. 
In order to check other reasons to explain this peculiar space charge behaviour, chemical measurements have been performed.

\section{Impact of e-beam irradiation on the chemical structure}

\subsection{Fourier Transform Infra-Red spectroscopy (FTIR)}

FTIR measurements, performed with the conditions described in section II-C, are presented in figure 8 for the reference and e-beam irradiated samples. In the case of the irradiated sample, the thickness is of $200 \mu \mathrm{m}$, in order to maximize the signal response of the irradiated zone compared to the non-irradiated one. From figure 8a, i.e. the complete spectra, there are not so much differences between the reference sample and the irradiated one, as no appearance or disappearance of a peak is observed. Looking carefully at some specific bands, such as the one at $1377 \mathrm{~cm}^{-1}$ related to $\mathrm{R}-\mathrm{CH}_{3}$ groups, i.e. end chains, this peak increases in the case of the irradiated sample, to a small extent (figure 8b) [20,21]. At the same time, there is a decrease of the $\mathrm{CH}_{2}$ groups, assigned to the linear aliphatic hydrocarbon chains $\left(1462 \mathrm{~cm}^{-1}\right)$ (figure $8 \mathrm{a}$ ). Another sign of the impact of the energy beam on the LDPE chemical structure is a decrease of the $-\mathrm{C}=\mathrm{O}$ peak at $1717 \mathrm{~cm}^{-1}$ which could indicate a decrease of ketone groups initially present in the insulation due to residual oxidation defects.
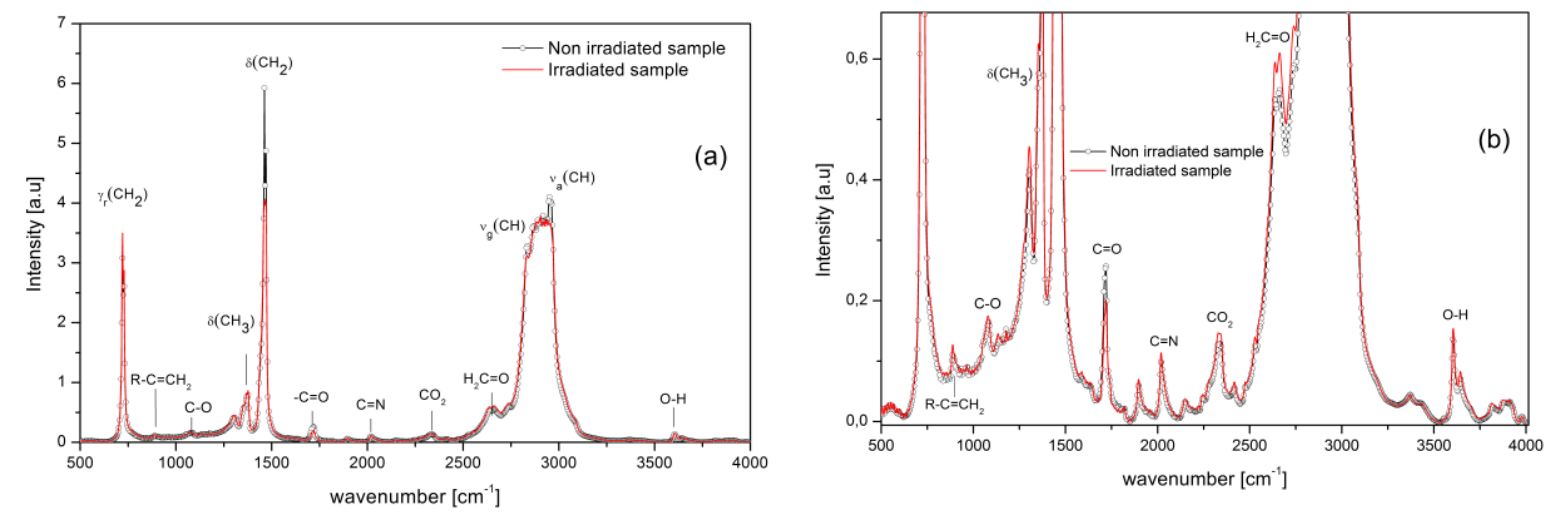

Figure 8. FTIR spectra (transmission mode) of a non-irradiated LDPE and an irradiated LDPE using a $80 \mathrm{keV}$ electron-beam with a current flux of $1 \mathrm{nA} / \mathrm{cm}^{2}$. (a) Total spectra view; (b) zoom.

Although a global observation of the infrared spectra does not show any new chemical function induced by the irradiation, some intensity differences between the irradiated and non-irradiated samples reveal a slight impact of the e-beam energy on the chemical structure of LDPE, notably the probable occurrence of chain scission. One can however wonder if this impact is sufficient to impact the material dielectric properties to the extent revealed by space charge measurements.

\subsection{Photoluminescence $(P L)$}

In order to confirm the presence of unsaturated groups revealed by FTIR measurements, PL measurements were performed on non-irradiated and irradiated LDPE samples, with an excitation wavelength range corresponding to the optical absorption of the polymer unsaturation. Figure 9 presents the PL spectra for an excitation wavelength ranging from $220 \mathrm{~nm}$ to $260 \mathrm{~nm}$. For both the irradiated and the non-irradiated LDPE sample, the maximum peak intensity is detected at $320 \mathrm{~nm}$ for an excitation wavelength of $230 \mathrm{~nm}$. The peak intensity is however weaker for the irradiated sample compared to the non-irradiated one. For the other excitation wavelengths, the emission peak remains centered around $320 \mathrm{~nm}$ with a small shift to high wavelength for the irradiated sample. This LDPE emission is known as the $\alpha, \beta$-unsaturated carbonyl groups of the enone and dienone type [22]. A second peak is also detected at $370 \mathrm{~nm}$ with excitation wavelength of $250 \mathrm{~nm}$ on the irradiated sample. Although this peak is not visible for the non-irradiated sample at this excitation wavelength, it seems present at 
$260 \mathrm{~nm}$, as the peak full width half maximum increases to a large extent. This peak has been already observed in the literature, but has been attributed till now to oxidation [23, 24]. As the non-irradiated LDPE is free from anti-oxidant, it is not unexpected that oxidation takes place. An observation of this peak for a lower excitation wavelength in the irradiated sample could be explained by an increase of this oxidation by the electron-beam.
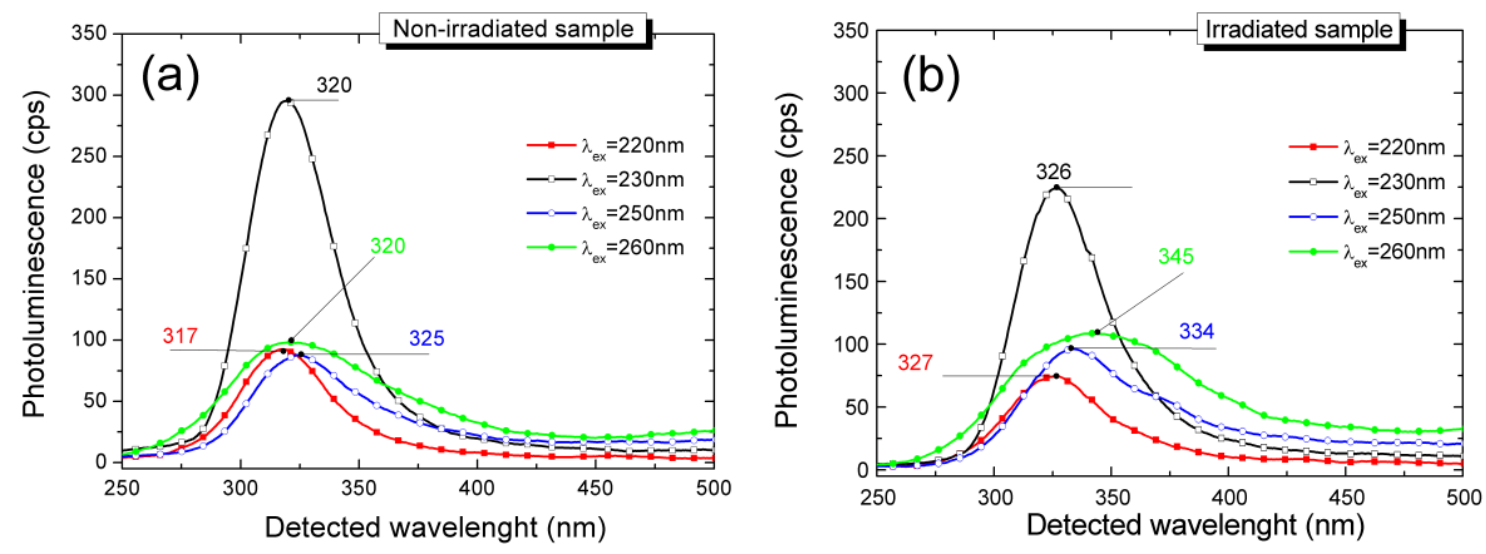

Figure 9. Photoluminescence spectrum of (a) non-irradiated LDPE and (b) e-beam irradiated LDPE, for different excitation wavelengths.

\section{Discussion}

Both the FTIR and the PL spectra realized under air conditions, after e-beam irradiation under vacuum, show some differences between an irradiated sample and a non-irradiated one.

The chemical impact does not seem as large as what has been published in the literature for such material irradiated under e-beam $[8,25]$. A fundamental parameter for the irradiation is the dose received by the material. The averaged dose deposited by the loss of energy of primary electrons has been estimated to $6 \mathrm{kGy}$, considering a homogeneous distribution over $80 \mu \mathrm{m}$ from the irradiation face. This is low comparatively to earlier results, pointing to the formation of trans-vinylene double bonds, carbonyl groups and crosslink nodes [8]. Doses above $20 \mathrm{kGy}$ were considered. In addition, the irradiations were achieved in air whereas our irradiations were achieved under high vacuum conditions. This can substantially change the chemistry at play owing to the presence of molecular oxygen in the ambient. Differential Scanning Calorimetry (DSC) has also been performed on our non-irradiated and irradiated specimens, and revealed a slight decrease (less than 3\%) of the crystallinity for the case of the irradiated sample compared to the non-irradiated one. All the chemical characterizations show some slight differences between the irradiated and the non-irradiated samples, large enough to be detectable, but not unequivocally proved to be a direct irradiation effect.

A main question to be solved is on the reversibility of the changes that are observed: if chemical damages have been produced and that these damages are at the origin of the changes in the space charge behaviour, then the effect should be permanent. If the effect is transitory, as due for example to the creation of electron-hole pairs, then it should decrease with elapsed time after irradiation. In order to address this aspect, a thermal treatment has been applied to an irradiated LDPE sample (same irradiation conditions as previously). The sample was kept in an oven at $50^{\circ} \mathrm{C}$ during 5 days under short-circuit. This kind of treatment is normally used to remove by-products from XLPE samples [26], or to remove charges from polymeric materials when already charged. With this thermal treatment, we expect to remove all the charges from the sample and evaluate if the LDPE behaves differently compared to the reference one, meaning that the irradiation has modified its chemical structure.

A space charge measurement was then performed on this LDPE sample, with the same protocol as the one described in section 2.2, and the result is presented in figure 10. During the $5 \mathrm{~min}$ of short-circuit 
before polarization, no charges are detected. Either the thermal treatment has removed the majority of charges, or the charge amount is so small that it is not detected by the PEA set-up. A last possibility is that the positive and negative charges are still in the insulation bulk, but at the same place (i.e. charges have been redistributed during the thermal treatment), showing a net zero charge distribution. During polarization, the space charge pattern is similar to the one obtained for a reference sample (i.e. figure 1), and substantially different from the ones obtained after irradiation (figure 4). Positive charges are detected in the whole material, not only in the irradiated zone. Moreover, the positive charges density is relatively low compared to the one observed on figure 4. During short-circuit, positive charges remain in the bulk while negative charges are observed close to the cathode. Therefore, it seems that the space charge features that are observed are essentially transitory. The observation, combined to the fact that the space charge seems symmetrical when considering the change in polarity of the applied voltage (figure 4 vs. figure 6) suggests that electron-hole pairs creation producing post radiation-induced conductivity effects are at play. The irradiated region has an apparent higher conductivity, and the space charge detected would be related to the gradient of conductivity setting up into the material.

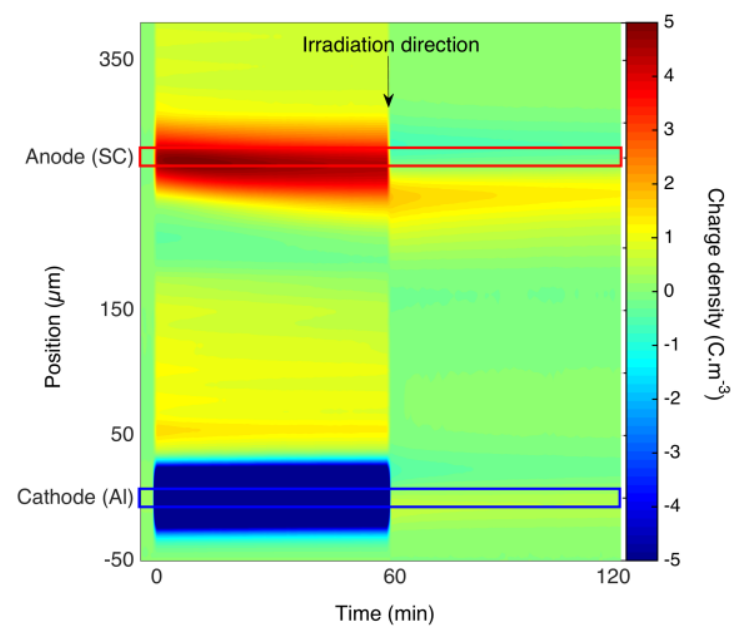

Figure 10. Cartography of space charge density for an e-beam irradiated LDPE film of $270 \mu \mathrm{m}$ thickness, at $25^{\circ} \mathrm{C}$, after 6 days under $50^{\circ} \mathrm{C}$. Short-circuit for 5 minutes; electric field of $40 \mathrm{kV} / \mathrm{mm}$ for one hour; and short-circuit for one hour. Irradiated zone next to the top electrode.

The main difference compared to Figure 1 is that these positive charges instantaneously appear in the bulk as soon as the electric field is applied. Negative charges, located at around $100 \mu \mathrm{m}$ from the irradiated face (top electrode) seem still present in a small amount, even after the thermal treatment. Possibly, a small amount of electron-hole pairs is still present even after thermal treatment. Under the effect of the field, positive charges would be quickly moved towards the cathode, leaving a net negative charge in the pre-irradiated region. An alternative explanation is that the electrons, generated by the beam, are very deeply trapped and are not easily removed. One could even think that these deep trapping levels, reached by the electron beam, cannot be filled by classical processes combining injection and transport. One of the questions that arise with this last measurement is if these deep traps have been created by the electron beam (i.e. associated with the slight changes in the chemical characterizations), or if these traps were already present before electron beam irradiation.

\section{Conclusion}

The electrical and physico-chemical behaviour of electron-beam irradiated LDPE have been investigated through this study.

Comparison of in-situ space charge measurements and ex-situ space charge measurements on the same LDPE samples irradiated using a $80 \mathrm{keV}$ electron beam of flux $1 \mathrm{nA} / \mathrm{cm}^{2}$ highlights the fact that positive charges are created within the sample during irradiation, due to the injection of charges at the grounded electrode and the creation of electron-holes pairs. These holes are however not observed with in-situ 
PEA measurements, but only with a 'classic' PEA set-up after e-beam irradiation, when the electric field distribution allows a rearrangement of the charge distribution.

Space charge measurements indicate the presence of positive and negative charges in irradiated LDPE films polarized under a DC electric field of $40 \mathrm{kV} / \mathrm{mm}$. Compared to the reference measurement performed on a non-irradiated LDPE sample, a large amount of charges are instantaneously detected in the whole irradiated zone, as soon as the electric field is applied. The charge density in the irradiated zone depends upon the applied electric field intensity whereas the sign of these charges depends upon the polarity of the applied electric field.

The peculiar behaviour of LDPE under applied voltage after e-beam irradiation is partially due to the presence of a large amount of charges (positive and negative) in the irradiated zone, that disturbs the electric field distribution and hence emphasizes the physical processes (conduction, injection, recombination ...) that are dominant in this region. One can however not neglect the chemical changes that could take place. Complementary FTIR and PL measurements report an increase of methyl groups possibly traducing chains scission, as well as a decrease of the carbonyl groups. The chemical changes seem however low compared to the literature on irradiated LDPE films, and compared to the changes in the dielectric response of the irradiated sample. It seems that the e-beam irradiation conditions are in the range where the chemical structure is beginning to be changed, but not enough to definitively state that the material has been aged.

More work needs to be performed to clearly assume that chemical degradation takes place: this will be performed experimentally, using different e-beam irradiation conditions, and also with the help of a fluid model, taking into account the different physical processes at play during irradiation and polarization. On the other hand, the weak chemical impacts, at this e-beam energy, would confirm that the charge implantation by electron beam is an alternative way to distinctly study generation and transport mechanisms in insulating materials.

\section{References}

[1] Dissado L A, Fothergill J C 1992 Electrical degradation and breakdown in polymers IET (London, United Kingdom), Chapter 9

[2] Montanari G C 2000 IEEE Trans. Dielectr. Electr. Insul. 7 309-315

[3] Labonte K 1982 IEEE Trans. Nucl. Sci. 29 1650-1653

[4] Sessler G M, Figueiredo M T and Ferreira G L 2004 IEEE Trans. Dielectr. Electr. Insul. 11 192-202

[5] Tomite N, Arai Y, Ohki Y, Nakmura H, Hinata T and Washio M 2013 Proc. IEEE Int. Conf. on Electrical Insulation and Dielectric Phenomena (Bologne, Italy) pp. 736-739

[6] Kikuchi Y, Kashiwagi S, Takada T, Miyake H and Tanaka Y 2013 Proc. IEEE Int. Conf. on Electrical Insulation and Dielectric Phenomena (Bologne, Italy) pp. 744-747

[7] Carrasco F, Pages P, Pascual S and Colom X 2001 Eur. Polym. J. 37 1457-1464

[8] Murray K A, Kennedy J E, McEvoy B, Vrain O, Ryan D, Cowman R and Higginbotham C L 2013 Nucl. Instr. Meth. Phys. Res. B. 297 64-74

[9] Griseri V, Malaval P, Berquez L, Tung T A, Le Roy S, Boudou L and Boulanger A 2010 Proc. Int. Conf. Electrical Insulation and Dielectric Phenomena (West Lafayette, USA), pp. 688-691

[10] Mary D, Albertini M and Laurent C 1997 J. Phys. D: Appl. Phys. 30 171-184

[11] Thomas C, Teyssedre G, Laurent C 2011 J. Phys. D: Appl. Phys. 44, 015401

[12] Tanaka Y, Chen G, Vaughan A and Takada T 2003 Proc. IEEE Int. Conference on Properties and Applications of Dielectric Materials (Nagoya, Japan) pp. 970-973

[13] Baro J, Sempau J, Fernández-Varea J M and Salvat F 1995 Nucl. Instr. Meth. Phys. Res. B 100 31-46

[14] Banda M E, Griseri V, Le Roy S and Teyssèdre G 2016 Proc. SFE Conference (Poitiers, France)

[15]Perrin C, Griseri V, Inguimbert C and Laurent C 2008 J. Phys. D: Appl. Phys. 41205417

[16]Le Roy S, Baudoin F, Griseri V, Laurent C and Teyssedre G 2012 J. Appl. Phys. 112023704

[17]Paulmier T, Dirassen B, Arnaout M, Payan D and Balcon N 2015 IEEE Trans. Plasma Sci. 43 2907-2914 
[18] Sim A. and Dennison J.R. 2013 5th AIAA Atmospheric and Space Environments Conference (San Diego, USA), AIAA-2013-2827 (31p)

[19] Montanari G C and Morshuis P H F 2005 IEEE Trans. Dielectr. Electr. Insul. 12 754-767

[20] Luongo J P and Salovey R 1963 J. Appl. Polym. Sci. 7 2307-2318

[21] Gulmine J V, Janissek P R, Heise H M and Akcelrud L 2002 Polymer Testing 21 557-563

[22] Konar J and Ghosh R 1990 J. Appl. Polym. Sci. 40 719-729

[23] Teyssedre G, Tardieu, G and Laurent C 2002 J. Mater. Sci. 37 1599-1609

[24] Jonsson J, Ranby B, Massines F, Mary D and Laurent C 1996 IEEE Trans. Dielectr. Electr. Insul. 3 859-865

[25] Khonakdar H A, Jafari S H, Wagenknecht U and Jehnichen D 2006 Radiat. Phys. Chem. 75 78-86

[26] Montanari G C, Laurent C, Teyssedre G, Campus A and Nilsson U H 2005 IEEE Trans. Dielectr. Electr. Insul. 12 438-446 